\title{
OSCILLATION OF LINEAR SECOND-ORDER DIFFERENTIAL SYSTEMS ${ }^{1}$
}

\author{
MAN KAM KWONG, HANS G. KAPER, KAZUO AKIYAMA AND \\ ANGELO B. MINGARELLI ${ }^{2}$
}

\begin{abstract}
This article is concerned with the oscillatory behavior at infinity of the solution $y:[a, \infty) \rightarrow \mathbf{R}^{n}$ of a system of second-order differential equations, $y^{\prime \prime}(t)+Q(t) y(t)=0, t \in[a, \infty) ; Q$ is a continuous matrix-valued function on $[a, \infty)$ whose values are real symmetric matrices of order $n$; it is assumed that the largest eigenvalue of the matrix $\int_{a}^{t} Q(s) d s$ tends to infinity as $t \rightarrow \infty$. Various sufficient conditions are given which guarantee oscillatory behavior at infinity; these conditions generalize those of Mingarelli [C.R. Math. Rep. Acad. Sci. Canada 2 (1980), 287-290, and Proc. Amer. Math. Soc. 82 (1981), 593-598].
\end{abstract}

1. Introduction. We are concerned with the differential equation

$$
y^{\prime \prime}(t)+Q(t) y(t)=0, \quad t \in[a, \infty),
$$

for a vector-valued function $y:[a, \infty) \rightarrow \mathbf{R}^{n}$. here $Q$ is a continuous matrix-valued function on $[0, \infty)$, whose values are real symmetric matrices of order $n$.

Two points $\alpha, \beta \in[a, \infty)$ are said to be conjugate relative to (1.1) if there exists a nontrivial function $y$ which satisfies (1.1) and vanishes at $\alpha$ and $\beta$. Equation (1.1) is said to be oscillatory at infinity if, for any point $\alpha \in[a, \infty)$, there exists a point $\beta \in(\alpha, \infty)$ such that $\alpha$ and $\beta$ are conjugate relative to (1.1). We use the notation $Q_{m}(t)$ to denote the matrix whose elements are the $m$ times repeated integrals over the interval $[a, t]$ of the corresponding elements of $Q(t)$ :

$$
Q_{0}(t)=Q(t) ; \quad Q_{m}(t)=\int_{a}^{t} Q_{m-1}(s) d s, \quad m=1,2, \ldots
$$

It has been conjectured (see Hinton and Lewis $[\mathbf{5}]$ ) that (1.1) is oscillatory at infinity whenever

$$
\lim _{t \rightarrow \infty} \lambda_{1}\left\{Q_{1}(t)\right\}=\infty,
$$

where $\lambda_{1}\{\cdot\}$ denotes the largest eigenvalue of the matrix inside the braces. This conjecture was studied recently by Mingarelli $[\mathbf{8}, \mathbf{9}]$, who proved that the oscillation

Received by the editors January 24, 1983.

1980 Mathematics Subject Classification. Primary 34A30, 34C10.

Key words and phrases. Matrix differential equation, oscillation theory, matrix Riccati equation. Riccati inequality.

${ }^{1}$ Work supported by the Applied Mathematical Sciences Research program (KC-04-02) of the Office of Energy Research of the U.S. Department of Energy under Contract W-31-109-ENG-38.

${ }^{2}$ This author wishes to acknowledge, with thanks, funding from the Natural Sciences and Engineering Research Council of Canada under Grant U0167. 
of (1.1) follows indeed from (1.3) provided either of the following two conditions is satisfied:

$$
\begin{aligned}
& \liminf _{t \rightarrow \infty} t^{-1} \operatorname{tr} Q_{1}(t)>-\infty, \\
& \liminf _{t \rightarrow \infty} t^{-1} \operatorname{tr} Q_{2}(t)>-\infty .
\end{aligned}
$$

Here, $\operatorname{tr}$ denotes the trace of the matrix. In this article we show that these conditions are stronger than necessary.

In $[\mathbf{8}, \mathbf{9}]$, as in the majority of work on systems of differential equations (cf., for example, Coppel [3, Chapter 2] or Reid [10, Chapter VII]), solutions of (1.1) are studied by means of the prepared or conjoined solutions of the associated matrix differential equation, i.e., solutions $Y(n \times n$ matrix-valued functions of $t)$ of the equation

$$
Y^{\prime \prime}(t)+Q(t) Y(t)=0, \quad t \in[a, \infty),
$$

for which $Y^{\prime}(t) Y^{-1}(t)$ is selfadjoint (Hermitian). Given a solution $Y$ of (1.6), one can define a matrix-valued function $R$ by the expression

$$
R(t)=-Y^{\prime}(t) Y^{-1}(t) .
$$

This function satisfies a Riccati equation of the form

$$
R(t)=R(a)+\int_{a}^{t} Q(s) d s+\int_{a}^{t} R^{2}(s) d s, \quad t \in[a, \infty) .
$$

In particular, if $Y$ is a conjoined solution of (1.6), then $R$ is a selfadjoint matrixvalued solution of (1.8), and vice versa. It can be shown that, if $R(a)$ is selfadjoint (real), then the matrix $R(t)$, which is uniquely determined by (1.8), is selfadjoint (real) for all $t$ for which it is defined. Of course, the matrix $R(t)$ may blow up at a finite value of $t$. This happens when the corresponding matrix $Y(t)$ becomes singular.

As Etgen and Pawlowski [4] have shown, (1.1) is nonoscillatory at infinity if and only if (1.6) has a nonsingular conjoined solution on $[a, \infty)$. (More precisely, (1.1) is nonoscillatory at infinity if and only if (1.6) has a nonsingular conjoined solution on $\left[a_{0}, \infty\right)$ for some $a_{0} \geq a$. However, as $a$ in (1.1) is arbitrary, there is no loss in generality if we take $a_{0}$ and $a$ to be the same point.) Hence, a necessary and sufficient condition for (1.1) to be nonoscillatory at infinity is that (1.8) has a continuous selfadjoint solution on $[a, \infty)$. It is the latter criterion that we shall use to study the oscillatory behavior of the system (1.1).

In the next section we show how the condition (1.3) leads to the Riccati inequality (2.14) for the scalar quantity $r(t)=\operatorname{tr} R(t)$. (In fact, any positive continuous linear functional on the space of real symmetric $n \times n$ matrices can be used instead of the trace to define the function $r$.) We then use recent results of Kwong and Zettl [6, 7] on the oscillation of scalar equations to establish a set of conditions weaker than (1.4) and (1.5) under which the condition (1.3) leads to oscillation of the system (1.1). Our main results are formulated in $\S 3$. 
2. A scalar Riccati inequality. We begin by rewriting (1.8) in the form

$$
R(t)=R(a)+Q_{1}(t)+\int_{a}^{t} R^{2}(s) d s, \quad t \geq a .
$$

By taking traces of both sides of the equation we obtain the scalar equation

$$
\operatorname{tr} R(t)=\operatorname{tr} R(a)+\operatorname{tr} Q_{1}(t)+\int_{a}^{t} \operatorname{tr} R^{2}(s) d s .
$$

Now suppose (1.3) holds, i.e.,

$$
\lim _{t \rightarrow \infty} \lambda_{1}\left\{Q_{1}(t)\right\}=\infty
$$

Then also $\lambda_{1}\left\{R(a)+Q_{1}(t)\right\} \rightarrow \infty$ as $t \rightarrow \infty$, and given $\varepsilon>0$ we can find a value $t_{0}$ such that

$$
\lambda_{1}\left\{R(a)+Q_{1}(t)\right\} \geq(1-\varepsilon) \lambda_{1}\left\{Q_{1}(t)\right\}, \quad t \geq t_{0} .
$$

In particular, by increasing $t_{0}$ if necessary, we can achieve that $\lambda_{1}\left\{R(a)+Q_{1}(t)\right\}$ is positive for all $t \geq t_{0}$. Furthermore, because the last term in (2.1) is nonnegative, we have the inequality

$$
\lambda_{1}\{R(t)\} \geq \lambda_{1}\left\{R(a)+Q_{1}(t)\right\}
$$

Hence,

$$
\operatorname{tr} R^{2}(t)=\sum_{i=1}^{n} \lambda_{i}^{2}\{R(t)\} \geq \lambda_{1}^{2}\{R(t)\} \geq(1-\varepsilon)^{2} \lambda_{1}^{2}\left\{Q_{1}(t)\right\}
$$

for all $t \geq t_{0}$. We use this estimate to derive an inequality for the scalar quantity $\operatorname{tr} R(t)$ from (2.2).

To this end we split the integral term in (2.2),

$$
\int_{a}^{t} \operatorname{tr} R^{2}(s) d s=\varepsilon \int_{a}^{t} \operatorname{tr} R^{2}(s) d s+(1-\varepsilon) \int_{a}^{t} \operatorname{tr} R^{2}(s) d s .
$$

Assuming $t \geq t_{0}$ and ignoring the contribution from the interval $\left[a, t_{0}\right]$ to the last integral, we estimate the remainder by means of (2.6),

$$
(1-\varepsilon) \int_{a}^{t} \operatorname{tr} R^{2}(s) d s \geq(1-\varepsilon)^{3} \int_{t_{0}}^{t} \lambda_{1}^{2}\left\{Q_{1}(s)\right\} d s .
$$

In the first integral in the right member of (2.7) we use the simple estimate

$$
\operatorname{tr} R^{2}(t) \geq \sum_{i=1}^{n} r_{i i}^{2}(t) \geq \frac{1}{n}(\operatorname{tr} R(t))^{2}
$$

to obtain the inequality

$$
\varepsilon \int_{a}^{t} \operatorname{tr} R^{2}(s) d s \geq \frac{\varepsilon}{n} \int_{a}^{t}(\operatorname{tr} R(s))^{2} d s
$$


Thus, combining the equations (2.2) and (2.7) with the inequalities (2.8) and (2.9), we find

$$
\begin{aligned}
\operatorname{tr} R(t) \geq & \operatorname{tr} Q_{1}(t)+\operatorname{tr} R(a)+(1-\varepsilon)^{3} \int_{t_{0}}^{t} \lambda_{1}^{2}\left\{Q_{1}(s)\right\} d s \\
& +\frac{\varepsilon}{n} \int_{a}^{t}(\operatorname{tr} R(s))^{2} d s .
\end{aligned}
$$

This inequality holds for all $t \geq t_{0}$. Since $\lambda_{1}\left\{Q_{1}(t)\right\} \rightarrow \infty$ as $t \rightarrow \infty$, the second term in the right member can be absorbed in the third term and the integral in the third term can be extended to an integral over the interval $[a, t]$. Thus

$$
\operatorname{tr} R(a)+(1-\varepsilon)^{3} \int_{t_{0}}^{t} \lambda_{1}^{2}\left\{Q_{1}(s)\right\} d s \geq(1-\varepsilon)^{4} \int_{a}^{t} \lambda_{1}^{2}\left\{Q_{1}(s)\right\} d s,
$$

provided $t$ is sufficiently large, $t \geq t_{1}$ say. Hence, if we let $\delta=(1-\varepsilon)^{4}$, replace $\varepsilon$ by $n \varepsilon$, and define

$$
\begin{gathered}
r(t)=\operatorname{tr} R(t), \quad t \geq a, \\
f(t)=\operatorname{tr} Q_{1}(t)+\delta \int_{a}^{t} \lambda_{1}^{2}\left\{Q_{1}(s)\right\} d s, \quad t \geq a,
\end{gathered}
$$

then $r(t)$ satisfies the scalar Riccati inequality

$$
r(t) \geq f(t)+\varepsilon \int_{a}^{t} r^{2}(s) d s
$$

for all $t \geq t_{1}$.

REMARK The inequality (2.14) remains valid if, instead of the trace, we use any positive continuous linear functional on the space of real symmetric $n \times n$ matrices to define the function $r$. Applying such a functional, $\phi$ say, to both sides of the equation (2.1), we obtain, instead of (2.2),

$$
\phi(R(t))=\phi(R(a))+\phi\left(Q_{1}(t)\right)+\int_{a}^{t} \phi\left(R^{2}(s)\right) d s .
$$

As shown by Akiyama [1], any continuous positive linear functional on the cone of nonnegative real symmetric matrices is equivalent with the trace functional. Hence, there exists a positive constant $c$, which depends only on $\phi$, such that $\phi\left(R^{2}(t)\right) \geq c \operatorname{tr}\left(R^{2}(t)\right)$. Instead of (2.6) we have therefore the inequality

$$
\phi\left(R^{2}(t)\right) \geq c(1-\varepsilon)^{2} \lambda_{1}^{2}\left\{Q_{1}(t)\right\}
$$

for all $t \geq t_{0}$. The analogue of $(2.7)$ is

$$
\int_{a}^{t} \phi\left(R^{2}(s)\right) d s=\varepsilon \int_{a}^{t} \phi\left(R^{2}(s)\right) d s+(1-\varepsilon) \int_{a}^{t} \phi\left(R^{2}(s)\right) d s .
$$

Here we use $(2.6)^{\prime}$ to estimate the last integral. In the first integral we use the elementary inequality

$$
\phi\left(R^{2}(t)\right) \geq(\phi(I))^{-1}(\phi(R(t)))^{2}
$$


where $I$ is the $n \times n$ identity matrix. Thus we obtain, instead of (2.10),

$$
\begin{aligned}
\phi(R(t)) \geq & \phi\left(Q_{1}(t)\right)+\phi(R(a))+c(1-\varepsilon)^{3} \int_{t_{0}}^{t} \lambda_{1}^{2}\left\{Q_{1}(s)\right\} d s \\
& +\frac{\varepsilon}{\phi(I)} \int_{a}^{t}(\phi(R(s)))^{2} d s .
\end{aligned}
$$

Now,

$$
\phi(R(a))+c(1-\varepsilon)^{3} \int_{t_{0}}^{t} \lambda_{1}^{2}\left\{Q_{1}(s)\right\} d s \geq c(1-\varepsilon)^{4} \int_{a}^{t} \lambda_{1}^{2}\left\{Q_{1}(s)\right\} d s
$$

for $t \geq t_{1}$, so if we let $\delta=c(1-\varepsilon)^{4}$, replace $\varepsilon$ by $\phi(I) \varepsilon$, and define

$$
\begin{gathered}
r(t)=\phi(R(t)), \quad t \geq a, \\
f(t)=\phi\left(Q_{1}(t)\right)+\delta \int_{a}^{t} \lambda_{1}^{2}\left\{Q_{1}(s)\right\} d s, \quad t \geq a,
\end{gathered}
$$

then (2.14) holds again.

Throughout the following analysis the trace functional can therefore be replaced by any positive linear functional $\phi$ on the space of real symmetric $n \times n$ matrices. This more general approach was taken by Akiyama $[\mathbf{1}]$ in his study of second order systems.

Inequalities of the type (2.14) have been studied extensively by Kwong and Zettl $[6,7]$ in connection with the oscillation theory of scalar second-order equations. These authors established several criteria (in terms of the function $f$ ) which are sufficient to show that the inequality $(2.14)$ is incompatible with the existence of a continuous function $r$ on the entire half-line. We mention only the following:

(i) There exists a measurable subset $J$ of $[a, \infty)$ with mes $J=\infty$, such that

$$
\lim _{t \in J, t \rightarrow \infty} f(t)=\infty \text {. }
$$

(ii) There exists a measurable subset $J$ of $[a, \infty)$ with mes $J<\infty$, such that (2.15) holds and

$$
\int_{J}(f(t))^{\gamma} d t=\infty
$$

for some $\gamma \in(0,1)$.

(iii) There exists a weight $w \in \mathcal{F}_{1}$ such that

$$
\liminf _{t \rightarrow \infty} \bar{f}_{w}(t)>-\infty
$$

where $\bar{f}_{w}$ is the weighted mean of $f$ relative to $w$,

$$
\bar{f}_{w}(t)=\int_{a}^{t} f(s) w(s) d s / \int_{a}^{t} w(s) d s, \quad t \geq a,
$$

and, furthermore,

$$
\lim _{t \rightarrow \infty} \int_{a}^{t}[f(t)-\lambda]_{+}^{2} d t=\infty
$$


for every $\lambda \in \mathbf{R}$, where $[x]_{+}=\max \{x, 0\}$. Here, $F_{1}$ is a family of weight functions introduced by Coles and Willett [2], which includes polynomial functions that are strictly positive on $[a, \infty)$.

(iv) There exists a weight $w \in \mathcal{F}_{0}$ such that

$$
\liminf _{t \rightarrow \infty} \bar{f}_{w}(t)>-\infty
$$

and

$$
\limsup _{t \rightarrow \infty} \bar{f}_{w}(t)=\infty
$$

Here, $\mathcal{F}_{0}$ is another family of weight functions introduced by Coles and Willett [2], which still includes polynomial functions that are strictly positive on $[a, \infty)$, but which is smaller than the family $\mathcal{F}_{1}$. (In [7] it was shown that this criterion is a special case of the previous criterion.)

In the next section we shall apply these criteria to obtain oscillation results for the system (1.1).

3. Main results. Our main result is contained in the following theorem.

THEOREM. Suppose (1.3) holds. Then (1.1) is oscillatory at infinity if, for some $\delta \in(0,1)$, the function $f$ defined in (2.13) satisfies one of the following conditions:

(i) The set $J=\{t \in[a, \infty): f(t) \geq 0\}$ has infinite measure.

(ii) The set $J=\{t \in[a, \infty]: f(t) \geq 0\}$ has finite measure and $\int_{J}(f(t))^{\gamma} d t=\infty$ for some $\gamma \in(0,1)$.

(iii) The conditions (2.17) and (2.18) hold for some $w \in \mathcal{F}_{1}$.

(iv) The conditions (2.19) and (2.20) hold for some $w \in \mathcal{F}_{0}$.

ProOF. Suppose (1.3) holds. Then either (1.1) is oscillatory at infinity or there exists a function $r$ which is defined and continuous on the half-line $[a, \infty)$, such that (2.14) holds for all sufficiently large $t$. The latter possibility is excluded by each of the criteria (i) through (iv).

Because $\lambda_{1}\left\{Q_{1}(t)\right\}$ will eventually be larger than any given constant, our result has the following weaker, but simpler, corollary.

COROLLARY 1. The theorem remains valid if the function $f$ is replaced by the function $f_{1}$,

$$
f_{1}(t)=\operatorname{tr} Q_{1}(t)+c t, \quad t \geq a,
$$

where $c$ is any positive constant.

To relate our results to those of Mingarelli $[8,9]$ we restate parts of Corollary 1.

COROLlaRY 2. Suppose (1.3) holds. Then (1.1) is oscillatory at infinity if, for some constant $c$,

$$
\operatorname{tr} Q_{1}(t) \geq-c t
$$

on a set of $t$ with infinite measure.

COROllaRY 3. Suppose (1.3) holds. Then (1.1) is oscillatory at infinity if

$$
\liminf _{t \rightarrow \infty} t^{-2} \operatorname{tr} Q_{2}(t)>-\infty .
$$


PROOF. If (3.3) holds, then there exists a constant $c>0$ such that

$$
t^{-1} \operatorname{tr} Q_{2}(t)+\frac{1}{4} c t \geq 0, \quad t \geq a .
$$

Hence,

$$
\frac{1}{t} \int_{a}^{t}\left(\operatorname{tr} Q_{1}(s)+c s\right) d s \geq \frac{1}{4} c t .
$$

The corollary follows from Corollary 1. (Take $w(t)=t$ in (2.19) and (2.20).)

COROLlary 4. Suppose (1.3) holds. Then (1.1) is oscillatory at infinity if

$$
\liminf _{t \rightarrow \infty} t^{-m-2} \operatorname{tr} \int_{a}^{t} s^{m} Q(s) d s>-\infty
$$

for some integer $m(m=1,2, \ldots)$.

Proof. The proof is the same as for Corollary 3. (Take $w(t)=t^{m}$.)

An example to which Corollary 2, but neither Corollary 3 nor Corollary 4, applies is given by $\operatorname{tr} Q_{1}(t)=t^{m} \sin t$, where $m>0$.

In conclusion, we mention that the same method can be used to investigate the oscillatory behavior of (1.1) at infinity if, instead of (1.3), one assumes that there exists a measurable set $J \subset[a, \infty)$ of infinite measure, such that

$$
\lim _{t \in J, t \rightarrow \infty} \lambda_{1}\left\{Q_{1}(t)\right\}=\infty .
$$

\section{REFERENCES}

1. K. Akiyama, On the maximum eigenvalue conjecture for the oscillation of second order differential systems, M.S. Thesis, University of Ottawa, 1983.

2. W. J. Coles and D. Willett, Summability criteria for oscillation of second order linear differential equations, Ann. Mat. Pura Appl. (4) 79 (1968), 391-398.

3. W. A. Coppel, Disconjugacy, Lecture Notes in Math., Vol. 220, Springer-Verlag, Berlin and New York, 1971.

4. G. J. Etgen and J. F. Pawlowski, Oscillation criteria for second order self-adjoint differential systems Pacific J. Math. 66 (1976), 99-110.

5. D. Hinton and R. T. Lewis, Oscillation theory for generalized second order differential equations, Rocky Mountain J. Math. 10 (1980), 751-766.

6. Man Kam Kwong and A. Zettl, Integral inequalities and second order linear oscillation, J. Differential Equations 45 (1982), 16-33.

7. __ Asymptotically constant functions and second order linear oscillation, J. Math. Anal. Appl. (to appear).

8. A. Mingarelli, An oscillation criterion for second order self-adjoint differential systems, C.R. Math. Rep. Acad. Sci. Canada 2 (1980), 287-290.

9. $\ldots$, On a conjecture for oscillation of second order ordinary differential sytems, Proc. Amer. Math. Soc. 82 (1981), 593-598.

10. W. T. Reid, Ordinary differential equations, Wiley, New York, 1971.

Mathematics and Computer Science Division, Argonne National laboraTORY, ARGONNE, Illinois 60439 (Current address of H. G. Kaper)

Department of Mathematics, University of Ottawa, Ottawa, Ontario K1N 9B4, CANADA (Current address of Kazuo Akiyama and A. B. Mingarelli)

Current address (M. K. Kwong): Department of Mathematics. Northern Illinois University, DeKalb, Illinois 60115 